\title{
Fracture of Device/Material
}

National Cancer Institute

\section{Source}

National Cancer Institute. Fracture of Device/Material. NCI Thesaurus. Code C63132.

Problem associated with a partial or full-thickness crack in the device materials. 